\title{
Bringing Entanglement to the High Temperature Limit
}

\author{
Fernando Galve, ${ }^{1}$ Leonardo A. Pachón, ${ }^{2}$ and David Zueco ${ }^{3}$ \\ ${ }^{1}$ IFISC (CSIC-UIB), Instituto de Física Interdisciplinar y Sistemas Complejos, Campus Universitat Illes Balears, \\ E-07122 Palma de Mallorca, Spain \\ ${ }^{2}$ Departamento de Física, Universidad Nacional de Colombia, Bogotá D.C., Colombia \\ ${ }^{3}$ Instituto de Ciencia de Materiales de Aragón y Departamento de Física de la Materia Condensada, \\ CSIC-Universidad de Zaragoza, E-50009 Zaragoza, Spain
}

(Received 19 March 2010; published 25 October 2010)

\begin{abstract}
Decoherence due to contact with a hot environment typically restricts quantum phenomena to the low temperature limit, $k_{B} T / \hbar \omega \ll 1$ ( $\hbar \omega$ is the typical energy of the system). Here we report the existence of a nonequilibrium state for two coupled, parametrically driven, dissipative harmonic oscillators which, contrary to generalized intuition, has stationary entanglement at high temperatures. This clarifies the role of temperature and could lighten the burden on quantum experiments requiring delicate precooling setups.
\end{abstract}

DOI: 10.1103/PhysRevLett.105.180501

Introduction.-The initial separation of microscopic quantum objects and macroscopic measuring apparatuses has been slowly demystified through careful scrutiny of which quantity it is that should be micro- or macroscopic. Neither size nor mass can be any more regarded as defining macroscopicity. Not even the amount of degrees of freedom forming the object, since interference fringes of massive many-atoms molecules [1], coherent superposition in Bose-Einstein condensates [2], and superconductivity have been observed. The role of quantum coherence in biological processes has been highlighted [3] for ambient temperatures, and recently, a proposal to create superpositions of dielectric bodies, such as viruses up to micron size, inside a high finesse optical cavity has been given [4]. However, demonstration of entanglement at high temperatures would be a much more extreme phenomenon.

It seems that the interaction with a hot environment, and thus the decoherence and relaxation of any object to the corresponding thermal state, is the only surviving classicality criterion. Put simply, if an object is subjected to an environment with a temperature exceeding the typical energy splitting, that is,

$$
k_{B} T>E_{\mathrm{typ}},
$$

we can be almost sure that it will quickly lose all its quantum features: superpositions and entanglement. We will show that, contrary to common intuition, this border drastically changes for nonequilibrium situations, and that indeed an entangled state between two interacting harmonic oscillators can be reached at any temperature, provided they can be driven according to the description given next.

We note that former proposals to entangle dissipative mirrors [5], membranes [6], and light modes [7], through driving, showed the feasibility of such schemes, but only for low temperatures. Entangling nanoresonators through parametric driven has also been proposed, though without analysis of decoherence mechanisms $[8,9]$. We sketch here
PACS numbers: 03.67.Bg, 03.65.Ud, 03.65.Yz, 05.70.Ln

a simple idea of how to produce an entangled nonequilibrium state at high temperatures. It may provide a huge leap in experimental requirements, while in addition it definitely relativizes the role of temperature in the list of possible criteria for classicality, the latter being an important theoretical topic. Furthermore, it supports the idea that we might expect entanglement in hot highly nonequilibrium situations, as pointed out [10] for biological systems.

The model and its solution.-In particular, we study the entanglement between two interacting identical harmonic oscillators. Though an idealization, it encompasses a reasonable description of a wide variety of objects in nature, in addition to artificial ones like nanomechanical oscillators [11], optical [12] and microwave cavities [13], and movable mirrors [14] to cite some, through which we expect to give a character of universality to the concepts that we expose here. The Hamiltonian of the system, $\mathcal{H}_{s}$ reads

$$
\mathcal{H}_{s}=\sum_{\alpha=1}^{2}\left(\frac{P_{\alpha}^{2}}{2 m}+\frac{1}{2} m \omega^{2} Q_{\alpha}^{2}\right)+c(t) Q_{1} Q_{2}
$$

with $m$ the mass of the oscillator, $\omega$ the frequency, and $c(t)$ the coupling coefficient. In what follows we assume

$$
c(t)=m c_{0}+m c_{1} \cos \left(\omega_{d} t\right)
$$

that is, we consider a time-dependent interaction, which plays a fundamental role in the creation and survival of entanglement. We explain later that the same effect can be obtained through modulation of the frequency, although a suboptimal efficiency is achieved.

In any realistic scenario the system is not completely isolated from the outside. The most rigorous way to include dissipation is by means of the system-bath model [15]. We couple the oscillators to two independent baths [see Fig. 1(a)],

$$
\mathcal{H}=\mathcal{H}_{s}+\sum_{\alpha, k=1}^{2, \infty} \frac{p_{\alpha, k}^{2}}{2 m_{k}}+\frac{m_{k} \omega_{k}^{2}}{2}\left(x_{\alpha, k}-\frac{c_{k} Q_{\alpha}}{m_{k} \omega_{k}^{2}}\right)^{2},
$$


where the baths are modeled by an infinite collection of harmonic oscillators [16]. This situation leads to thermalization and decoherence for all degrees of freedom [17], unlike the common bath case [18]. Besides, we avoid extra correlations between parties induced by the common bath [19].

The evolution for the density matrix of the two oscillators, $\rho_{s}$, can be cast as,

$$
\rho_{s}\left(X_{f}, t\right)=\int d^{4} X_{i} J\left(X_{f}, t ; X_{i}, 0\right) \rho_{s}\left(X_{i}, 0\right),
$$

with $X=\left\{Q_{1,+}, Q_{1,-}, Q_{2,+}, Q_{2,-}\right\}$ and $J\left(X_{f}, t ; X_{i}, 0\right)$ being the influence functional which is given in terms of a path integral expression after tracing out the environmental degrees of freedom [20].

Usually, the analytical evaluation of $J\left(X_{f}, t ; X_{i}, 0\right)$, even for time-independent systems, is only possible in very few cases $[16,21]$. Here, we have been able to derive an exact analytic expression for $J\left(X_{f}, t ; X_{i}, 0\right)$ [22]. We assumed for simplicity an Ohmic spectral density $I(\omega) \hat{=} \sum_{j} c_{j}^{2} /$ $\left(2 m_{j} \omega_{j}\right) \delta\left(\omega-\omega_{j}\right)=m \gamma \omega$, which produces white noise in the classical limit [21]. Our exact result allows studying any regime (low or high temperature, strong or weak damping, etc.) without approximations, so any system
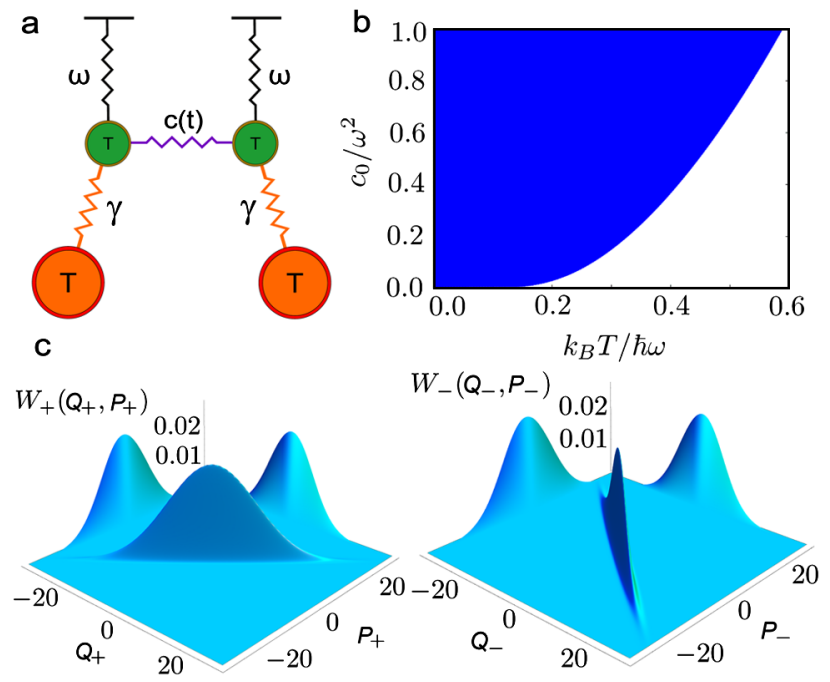

FIG. 1 (color online). (a) The system is formed by two linearly coupled oscillators, initially thermalized due to each of them being dissipatively coupled to an environment at temperature $T$. Driving the coupling (or frequency) sinusoidally leads to the production of entanglement even at very high temperatures. (b) Entanglement phase diagram for the case without driving. The state thermalizes to a state with no entanglement (white) unless the temperature is below the quantum limit $k_{B} T<\hbar \omega$. We used $\gamma=0.0005 \omega$. (c) Wigner phase space representation of the normal modes. They are sufficiently squeezed along orthogonal directions, so the oscillators are entangled $\left(E_{N} \simeq 0.7\right)$. The parameters are $k_{B} T / \hbar \omega=5, \gamma=0.005 \omega, c_{1}=0.5 m \omega^{2}$, while the snapshot has been taken at time $\omega t=12.5$. The projections have been drawn to guide the eye. that can be considered as two harmonic oscillators with linear coupling can be ascribed exactly to our description.

Linearity of the total Hamiltonian ensures that the state is always Gaussian, and thus its entanglement properties are fully characterized by the covariance matrix $\sigma_{i, j}=$ $\left\langle R_{i} R_{j}+R_{j} R_{i}\right\rangle / 2-\left\langle R_{i}\right\rangle\left\langle R_{j}\right\rangle$ with $R=\left(Q_{1}, Q_{2}, P_{1}, P_{2}\right)$, which can be calculated from the propagator $J\left(X_{f}, t\right.$; $\left.X_{i}, 0\right)$. A computable measure of entanglement is known for Gaussian states, the logarithmic negativity $E_{N}$ [23].

Entanglement in the time-independent case. - In the absence of driving [ $c_{1}=0$ in (3)], contact with an environment asymptotically forces each particle into a thermal state with a temperature equal to that of the bath it is connected to. This state is reached independently on the initial condition of the oscillator and leads to the entanglement characteristics shown in Fig. 1(b). That is, any state will, after thermalization, fall into either the shaded (blue, entangled) part or the white (separable) part, depending on the ratio $c_{0} / \omega^{2}$ and the bath's temperature [17]. Notice that at weak dissipation strength, the equilibrium phase diagram is mostly independent on $\gamma$ [24]. Above the so-called quantum limit $k T<\hbar \omega$ [25], as expected from intuition, each oscillator has an independent description because the quantum state is separable (we focus on entanglement since it underlies the very heart of the quantum weirdness).

Entanglement creation by driving.-The normal mode transformation for the oscillator Hamiltonian (2) reads $\tilde{H}=\sum_{\alpha= \pm} P_{\alpha}^{2} / 2 m+m \omega_{ \pm}^{2} Q_{\alpha}^{2} / 2 \quad$ where $Q_{ \pm}=\left(Q_{1} \pm\right.$ $\left.Q_{2}\right) / \sqrt{2}\left[P_{ \pm}=\left(P_{1} \pm P_{2}\right) / \sqrt{2}\right]$ and $\omega_{ \pm}^{2}=\omega \pm c(t) / m$. In the continuous variable setting, it is known that the maximally entangled state is the Einstein-Podolsky-Rosen wave function [26]. It is just the infinite squeezing limit of the two-mode squeezed vacuum state, in which the indeterminacies of $Q_{+}$and $P_{-}$are under the standard quantum limit set by Heisenberg's principle, while $Q_{-}$and $P_{+}$are above it [such that $\Delta Q_{ \pm} / \Delta P_{ \pm}=\exp (\mp 2 r) / \omega^{2}$, with $r$ the so-called squeezing parameter]. The opposite situation is also valid. Thus generation of entanglement can be provided by squeezing of the normal modes, which in turn can be generated through parametric driving of their frequencies $[8,9]$. Both a time dependence in $\omega$ or $c$ will do; however, the latter is better because it naturally provides the correct combination of squeezing between \pm modes [9]. At the same time, the environment will try to destroy quantum coherence through equilibration to the thermal state. The balance between these two competing effects will determine whether the state has stationary entanglement or not. In Fig. 1(c) we provide an example of normal mode squeezing in the presence of the bath above the typical quantum limit (1) $k_{B} T / \hbar \omega=5>1$.

In Fig. 2 we summarize our results. Indeed, we find sets of parameters where entanglement is present at temperatures beyond the quantum limit; notice that in both figures $k_{B} T>\hbar \omega$. Starting with a thermal state at the bath's temperature, the system reaches after a certain time a nonequilibrium state with nonzero stationary entanglement. We 
have chosen rather conservative couplings to the baths, and still very high temperatures, $k_{B} T \gg \hbar \omega$, can be reached.

The highly nonequilibrium state into which the system is forced reaches the same amount of stationary entanglement, independent on the initial state of the system. To show this effect we plot in Fig. 3 (see inset) the time evolution of entanglement when the system starts with a two-mode squeezed state and squeezing parameters $r=0$, 0.5 , and 1 , and compare it to the case of an initial thermal state with the same temperature as the bath.

New phase diagram for entanglement.-Parametric driving yields a new asymptotic behavior which defines a new "phase diagram," now dependent on four parameters: driving amplitude, frequency, temperature, and the coupling to the bath. The driving frequency is overall chosen to be $\omega_{d}=2 \times 0.998 \omega$, and we also set $c_{0}=0$. While the optimal squeezing generation is obtained with a $\omega_{d}$ dependent on $\omega$ and $c_{1}$, this value of $\omega_{d}$ seems to produce results nearly as good for different parameters, so it will be used unless otherwise stated. In Fig. 3 we see the points which delimit the border between presence (left) or absence (right) of entanglement, which is linear in temperature and driving amplitude and, as expected, the more isolated and driven the system is (low $\gamma$ and high $c_{1}$ ), the higher the temperature can be reached. In addition to the exact result, we have plotted a simple estimation of the border which we explain next.
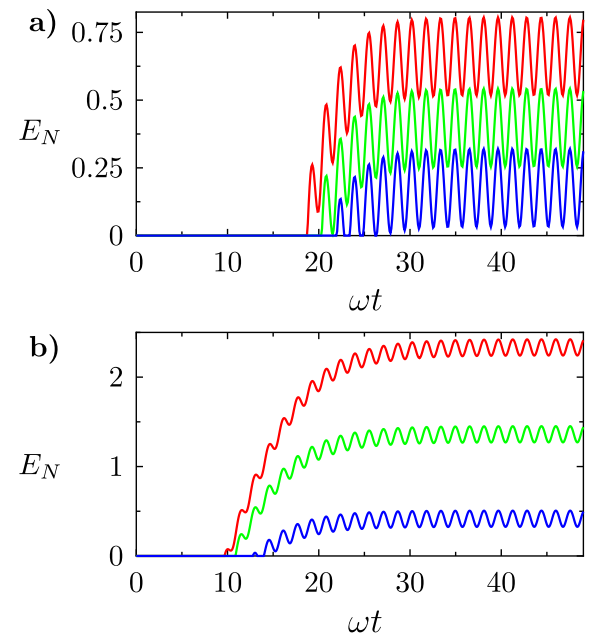

FIG. 2 (color online). Time evolution of entanglement towards its stationary value at (a) different environmental temperatures $k_{B} T / \hbar \omega=250$ [grey (red)], 300 [light grey (green)], and 350 [dark grey (blue)], with a damping of $\gamma=0.005 \omega$, driving amplitude of $c_{1}=0.5 m \omega^{2}$, and driving frequency $\omega_{d}=2 \times$ $0.998 \omega$. The stationary regime is reached in a reasonable time with a significative amount of entanglement. Notice that for an oscillator with frequency $\omega=21 \mathrm{GHz}$ the values of temperature are directly given in Kelvin, and this would imply observation of entanglement at room temperature. (b) Now the temperature is kept fix, $k_{B} T / \hbar \omega=5$, with the same parameters, while the damping parameter is varied: $\gamma=0.005 \omega$ [grey (red)], $0.01 \omega$ [light grey (green)], $0.02 \omega$ [dark grey (blue)].

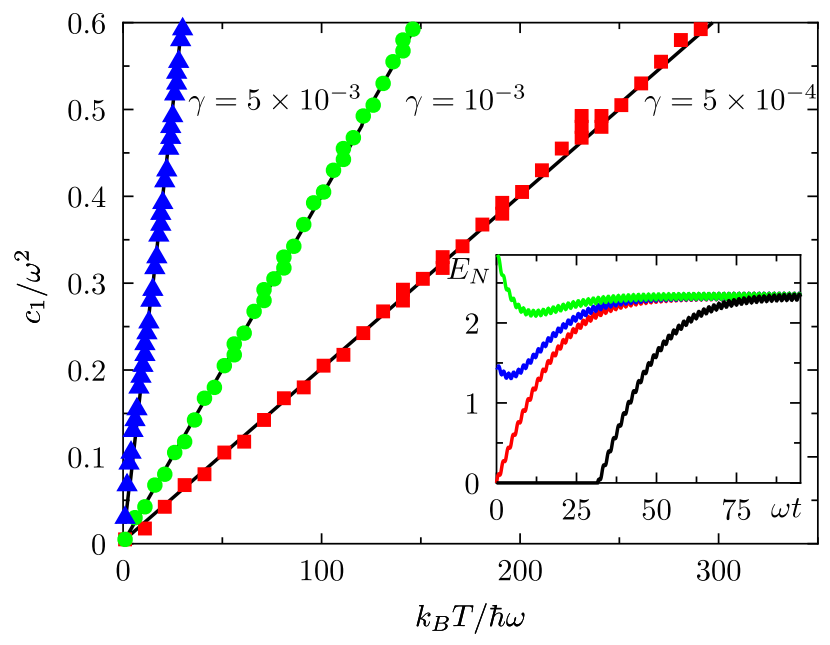

FIG. 3 (color online). Phase diagram of entanglement in the presence of parametric driving. We compare the condition (6) [lines] with the exact time evolution [dots] for different bath couplings $\gamma=0.005 \omega$ [dark grey (blue) triangles], $0.001 \omega$ [light grey (green) circles] and $0.0005 \omega$ [grey (red) squares]. Inset: time evolution for different initial conditions, namely, a two-mode squeezed vacuum state (dotted curves) with squeezing parameter $r=0$ [grey (red)], 0.5 [dark grey (blue)], and 1 [light grey (green)], as compared to that of an initial thermal state (black). They all converge after some tens of periods. The parameters here are $\gamma=0.001 \omega, c_{1}=0.2 \omega^{2}, \quad \omega_{d}=2 \times$ $0.998 \omega$, and $k_{B} T / \hbar \omega=10$.

The rate of squeezing (leading to entanglement) can be obtained from the solutions to the nondissipative driven problem, having the Mathieu form $x(t)=\exp \left(i \mu_{M} t\right) \phi(t)$, where $\phi(t)$ is a periodic function. If the Mathieu characteristic exponent $\mu_{M}$ is real, they are stable, otherwise they are divergent which implies production of squeezing at a rate $\left|\operatorname{Im}\left(\mu_{M}\right)\right|$ (for every damped solution there is a divergent one) [27]. The exponent $\left|\operatorname{Im}\left(\mu_{M}\right)\right|$ equals $c_{1} / 4 \omega^{2}$ when $\omega_{d} \simeq 2 \omega$ and $c_{1} / \omega^{2}$ is small. The rate of decoherence can be estimated from the diffusion coefficient $D_{p p} \simeq$ $m \gamma k_{B} T$ [28]. Thus by comparison of both rates we obtain the new condition under which entanglement is present:

$$
\frac{k_{B} T}{\hbar \omega} \leq \frac{\left|\operatorname{Im}\left(\mu_{M}\right)\right|}{\gamma},
$$

which is seen to be a rather impressive match to the exact evolution (see Fig. 3). The condition above should be compared with the standard condition (1). In a nutshell the driving brings in a new quantum limit.

Some examples.-We give next some actual examples of experiments which could profit from our strategy. However, an additional comment is in order: the fact that squeezing grows approximately as $\left|\operatorname{Im}\left(\mu_{M}\right)\right| t$ also means that the energy and delocalization in space are increasing exponentially in time. Thus checking consistency with experimental size and energy considerations is a must.

Take, for example, two calcium ions, each confined in its own planar Penning traps [29]. A trap can be fabricated by 
nanolithography with a size of $d \sim 0.12 \mu \mathrm{m}$. If a voltage of $V=10 \mathrm{~V}$ is applied, the motional frequency is $\omega \simeq$ $21 \mathrm{GHz}$ and thus we can interpret Fig. 3 as the temperature in Kelvin. A wire mediated capacitive coupling between traps allows us to reduce the effective distance between ions and makes the coupling increase up to a reasonable level $c(t) / m=c_{0}=0.047 \omega_{0}^{2}$. If the frequencies are driven instead of the coupling [i.e., $\omega(t)=\omega_{0}+$ $\left.\omega_{1} \sin \omega_{d} t\right)$ ], and assuming $\gamma=0.0005 \omega$, we still manage to get entanglement up to $\sim 50 \mathrm{~K}$, while the delocalization of the oscillators is yet below the trap size, ensuring no confinement leakage. To reach room temperature, a very strong coupling would be required indeed, but our method allows the experimentalist to avoid building a sub- $4 \mathrm{~K}$ (colder than liquid helium) setup. We believe this to be a huge experimental step.

Another example is microwave superconducting cavities [30]. Their coupling can be modulated by placing a superconducting qubit between them. The typical frequencies in these resonators are in the $\mathrm{GHz}$ regime, operating usually in the millidegrees Kelvin range. The decoherence in these systems is $\gamma \cong 10^{-4} \omega$, or even less. However, the coupling is weak, around $10 \mathrm{MHz}$. In this case, due to the weak coupling, the parametric driving would enhance the amount entanglement that could be measured by presentday technology [31].

Current experiments with nanomechanical resonators have these typical parameters: $\omega=2 \pi \nu=2 \pi \times 15 \mathrm{MHz}$, $m=10^{-17} \mathrm{~kg}, c_{1} \sim 10^{-3} \omega^{2}$, and a quality factor $Q \sim$ 20000 , which yields a damping $\gamma=5 \times 10^{-5} \omega$ [32]. Steady entanglement can be observed at tens of millidegrees Kelvin. If the frequency of our coupling can be increased by a factor 10, it might reach the entangled regime in the range of Kelvin.

All these examples together with recent proposals and experimental achievements, such as strong coupling [12] and parametric driving of the coupling [8], make it feasible that in the near future we might measure entanglement in yet unsuspected temperature regimes, while eliminating the need for complex and costly setups to cool objects to the quantum regime.

We acknowledge Peter Hänggi and Gert-Ludwig Ingold for enlightened discussions and advice. We also thank the Universität Augsburg where this work was started. D. Z. acknowledges financial support from FIS2008-01240 and FIS2009-13364-C02-01 (MICINN), F. G. from COQUSYS (200450E566) (IFISC-CSIC), and L.A.P. from Colciencias and the Universidad Nacional de Colombia.
[1] K. Hornberger et al., New J. Phys. 11, 043032 (2009).

[2] M. R. Andrews et al., Science 275, 637 (1997).

[3] E. Collini et al., Nature (London) 463, 644 (2010).

[4] O. Romero-Isart et al., New J. Phys. 12, 033015 (2010).

[5] D. Vitali, S. Mancini, and P. Tombesi, J. Phys. A 40, 8055 (2007).

[6] M. J. Hartmann and M. B. Plenio, Phys. Rev. Lett. 101, 200503 (2008).

[7] M. B. Plenio and S. F. Huelga, Phys. Rev. Lett. 88, 197901 (2002).

[8] L. Tian, M. S. Allman, and R. W. Simmonds, New J. Phys. 10, 115001 (2008).

[9] F. Galve and E. Lutz, Phys. Rev. A 79, 032327 (2009).

[10] J. Cai, S. Popescu, and H. J. Briegel, Phys. Rev. E 82, 021921 (2010).

[11] K. C. Schwab and M.L. Roukes, Phys. Today 58, 36 (2005).

[12] A. Gröblacher et al., Nature (London) 460, 724 (2009).

[13] A. Wallraff et al., Nature (London) 431, 162 (2004).

[14] W. Marshall et al., Phys. Rev. Lett. 91, 130401 (2003).

[15] U. Weiss, Quantum Dissipative Systems (World Scientific, Singapore, 1993).

[16] A. O. Caldeira and A. L. Leggett, Ann. Phys. (N.Y.) 149, 374 (1983).

[17] K. L. Liu and H. S. Goan, Phys. Rev. A 76, 022312 (2007).

[18] J. P. Paz and A. J. Roncaglia, Phys. Rev. Lett. 100, 220401 (2008).

[19] O. S. Duarte and A. O. Caldeira, Phys. Rev. Lett. 97, 250601 (2006).

[20] R. P. Feynman and J. Vernon F. L., Ann. Phys. (N.Y.) 24, 118 (1963).

[21] H. Grabert, P. Schramm, and G. L. Ingold, Phys. Rep. 168, 115 (1988).

[22] See supplementary info at http://link.aps.org/supplemental/ 10.1103/PhysRevLett.105.180501 for the derivation of the complete solution.

[23] G. Vidal and R. F. Werner, Phys. Rev. A 65, 032314 (2002).

[24] P. Hänggi and G.-L. Ingold, Chaos 15, 026105 (2005).

[25] In the case of coupled oscillators the typical energy splitting $E_{\text {typ. }}$ of Eq. (1) is given approximately by $\hbar \omega$, when $c_{0}$ is small; see J. Anders and A. Winter, Quantum Inf. Comput. 8, 0245 (2008).

[26] A. Einstein, B. Podolsky, and N. Rosen, Phys. Rev. 47, 777 (1935).

[27] C. Zerbe and P. Hänggi, Phys. Rev. E 52, 1533 (1995).

[28] W. Zurek, Rev. Mod. Phys. 75, 715 (2003).

[29] S. Stahl et al., Eur. Phys. J. D 32, 139 (2005).

[30] M. Mariantoni et al., Phys. Rev. B 78, 104508 (2008); G. M. Reuther et al., ibid.81, 144510 (2010)

[31] E. P. Menzel et al., Phys. Rev. Lett. 105, 100401 (2010).

[32] M. J. Woolley, G. J. Milburn, and C. M. Caves, New J. Phys. 10, 125018 (2008). 